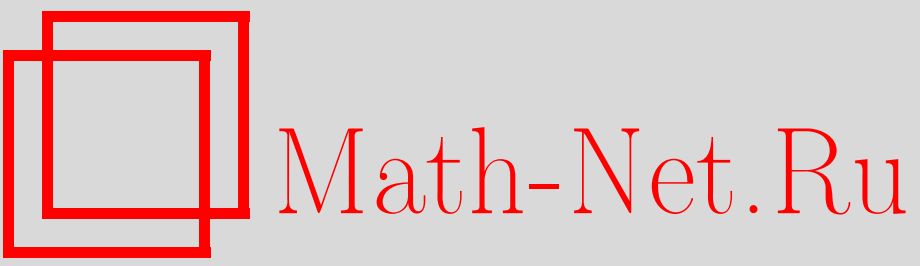

И. Д. Манджавидзе, А. Н. Сисакян, Теория возмущений в окрестности протяженных объектов, ТМФ, 2000, том 123, номер 3, 433-451

DOI: https://doi.org/10.4213/tmf614

Использование Общероссийского математического портала Math-Net.Ru подразумевает, что вы прочитали и согласны с пользовательским соглашением

http://www.mathnet.ru/rus/agreement

Параметры загрузки:

IP : 54.205 .225 .156

26 апреля 2023 г., 10:14:36 
ТЕОРЕТИЧЕСКАЯ

И МАТЕМАТИЧЕСКАЯ

ФИЗИКА

Том 123, № 3

июнь, 2000

(C) $\mathbf{2 0 0 0}$ r.

И. Д. Манджавидзе ${ }^{* \dagger}$, А. Н. Сисакян ${ }^{\dagger}$

\section{ТЕОРИЯ ВОЗМУЩЕНИЙ В ОКРЕСТНОСТИ ПРОТЯЖЕННЫХ ОБЪЕКТОВ}

С помощью унитарного отображения к переменным "действие-угол" сформулирована теория возмущений по обратной константе взаимодействия в окрестности нетривиального экстремума действия. Описана также обычная теория возмущений в этой окрестности.

Работа посвящается светлой памяти М. В. Савельева

\section{1. ВВЕДЕНИЕ}

Рассматриваемая в данной работе проблема квантования протяженных объектов типа солитонов имеет более чем двадцатилетнюю историю (см., например, обзорные статьи $[1,2])$. Отсутствие прогресса в ее решении вызывает беспокойство, если вспомнить о множестве важных физических задач, с которыми она связана.

Одно из возможных решений рассматриваемой проблемы предложено в работе [3]. Цель нашей статьи - показать, что описанный в работе [3] подход приводит к теории возмушений в случае сильной связи, когда разложение ведется по степеням $1 / g$, где $g$ константа взаимодействия.

Наш подход основывается на идее работы [4], что функциональная мера $D M$ интегрального представления для функции

$$
\rho(E)=\int d u_{1} d u_{2}\left|\left\langle u_{2} ; E \mid u_{1} ; E\right\rangle\right|^{2}
$$

$\delta$-образна (дираковская мера), т.е.

$$
D M(u)=\prod_{t} d u(t) \delta\left(\frac{\delta S(u)}{\delta u(t)}+j(t)\right),
$$

где $j(t)$ - случайная сила квантовых возмущений. Мы будем рассматривать простейшую квантово-механическую задачу. Состояния $\left|u_{i} ; E\right\rangle$ в формуле (1.1) описываются

* Институт физики, Тбилиси, Грузия. E-mail: joseph@nusun.jinr.ru

$\dagger$ Объединенный институт ядерных исследований, Дубна, Россия 
граничными значениями координаты $u_{i}$ и энергией $E$ частицы. В п. 2.1 будет описан физический смысл меры (1.2), ее получение будет дано в п. 2.2.

Наличие $\delta$-функции в выражении (1.2) означает, что точное локальное равенство

$$
-\frac{\delta S(u)}{\delta u(t)}=j(t)
$$

определяет полный набор необходимых вкладов. Общие свойства теории на $\delta$-образной мере приведены в п. 2.3. Следует подчеркнуть, что равенство (1.3) не есть следствие гамильтонова вариационного принципа и мера (1.2) будет построена с помощью принципа сохранения полной вероятности (условия унитарности) [4].

Равенство (1.3) указьвает на то, что преобразование кинетической части лагранжиана индуцирует касательное преобразование квантового источника $j(t)[5]$. Мы воспользуемся этим, чтобы описать квантовую динамику в удобных нам терминах. А именно будет рассмотрено каноническое преобразование меры (1.2), позволяющее перейти к коллективным переменным протяженных объектов (в теории поля это будут, например, координаты и импульсы солитонов). Эти переменные имеют смысл переменных “действие-угол" и образуют кокасательное расслоение $T^{*} \Omega$ исходного фазового пространства $\Omega$. Таким образом, используемое в данной работе преобразование является обычным в классической механике "отображением момента" [6]:

$$
J:(u, p) \rightarrow(\xi, \eta)
$$

где $p$ - сопряженньй $u$ импульс (см. ниже п. 4.1 и п. 4.2). По этой причине будет использована именно гамильтонова формулировка механики.

Мы рассмотрим квантовую механику, предполагая, что потенциал имеет вид

$$
v(u ; g)=\frac{1}{g} v\left(g^{1 / 2} u ; 1\right) \equiv \frac{1}{g} v\left(g^{1 / 2} u\right),
$$

для простоты предполагается, что функция $v(u)$ отвечает потенциальной яме с одним

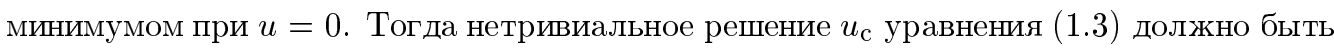
сингулярно при $g=0$, т.е.

$$
u_{\mathrm{c}}=O\left(g^{-1 / 2}\right),
$$

по крайней мере в низших порядках по $j$.

Более того, мы найдем, что ряд преобразованной теории возмушений представляет собой разложение по степеням $1 / g$ и коэффициенты разложения вычисляются достаточно просто (поскольку расслоение $T^{*} \Omega$ - однородное и изотропное пространство).

Предлагаемая теория возмушений кардинально отличается от ранее сформулированной теории возмушений в случае слабой связи $[1,2,7]$. В разделе 3 эта теория возмущений по константе $g$ будет выведена с использованием меры (1.3), что позволяет явно показать причину появления разложения по степеням $1 / \mathrm{g}$. Строго говоря, нет никакой связи между коэффициентами разложения обеих теорий возмущений. Однако оба разложения должны существовать одновременно в том смысле, что мы можем использовать 
разложение или по степеням $g$, или по степеням $1 / g$. В этом смысле оба разложения дуальны друг другу (см. раздел 5). Преимушество разложения по степеням $1 / \mathrm{g}$ заключается в том, что мы можем в этом случае провести все вычисления до конца (см. п. 2.3 и п. 4.2).

В разделе 2 выведено интегральное представление для $\rho$ с мерой (1.2). В разделе 3 описана теория возмущений в случае слабой связи. В разделе 4 рассмотрено отображение (1.4) для квантовой системы, получено разложение по степеням $1 / g$ и указаны правила вычисления коэффициентов этого разложения. В разделе 5 предложена качественная интерпретация построенной теории возмушений.

\section{2. УНИТАРНОЕ ОПРЕДЕЛЕНИЕ МЕРЫ}

Вначале мы объясним роль условия унитарности в определении функциональной меры (п. 2.1). Далее будет показано, как вариационный принцип Даламбера может быть выведен для квантовых систем (п. 2.2), и, наконец, мы опишем общие свойства теории на $\delta$-образной мере.

2.1. Формулировка метода. Чтобы вычислить энергию связанных состояний $E_{n}$, достаточно рассмотреть след [8]

$$
R(E)=\sum_{n} \int d u \frac{\psi_{n}(u) \psi_{n}^{*}(u)}{E-E_{n}-i \varepsilon}=\sum_{n} \frac{1}{E-E_{n}-i \varepsilon}=\operatorname{Tr} \frac{1}{E-\mathbf{H}-i \varepsilon}
$$

где $\mathbf{H}$ - оператор Гамильтона, $\varepsilon \rightarrow+0$ и зависимость от волновых функций $\psi_{n}$ исчезла в силу их ортонормируемости.

Квазиклассическое приближение дает [8]

$$
R(E) \sim \sum_{k=0}^{\infty} e^{i k\left(S_{1}\left(u_{\mathrm{c}}\right)-\pi\right)}=\frac{1}{1+e^{i S_{1}\left(u_{\mathrm{c}}\right)}}
$$

где $S_{1}\left(u_{\mathrm{c}}\right)$ - действие за время элементарного цикла (один период) на замкнутой траектории $u_{\mathrm{c}}=u_{\mathrm{c}}(E)$. Положение полюсов в формуле $(2.2)$ определяет значения $E_{n}$.

Отметим теперь, что в силу соотношения

$$
\frac{1}{E-E_{n}-i \varepsilon}=\mathrm{P} \frac{1}{E-E_{n}}+i \pi \delta\left(E-E_{n}\right) \text { при } \varepsilon=0
$$

нам нет необходимости вычислять действительную часть, т.к. она не содержит измеримого значения энергии $E_{n}$,

$$
\mathrm{P} \frac{1}{E-E_{n}}=0 \text { при } E=E_{n} .
$$


По этой причине, следуя работе [4], мы будем вычислять значительно более простую величину

$$
\begin{aligned}
\varepsilon \rho(E) & =\varepsilon \sum_{n_{1}, n_{2}} \int d x_{1} d x_{2} \frac{\psi_{n_{1}}\left(x_{1}\right) \psi_{n_{1}}^{*}\left(x_{2}\right)}{E-E_{n_{1}}-i \varepsilon} \frac{\psi_{n_{2}}^{*}\left(x_{1}\right) \psi_{n_{2}}\left(x_{2}\right)}{E-E_{n_{2}}+i \varepsilon}= \\
& =\varepsilon \sum_{n}\left|\frac{1}{E-E_{n}-i \varepsilon}\right|^{2}=\frac{1}{2 i} \sum_{n}\left\{\frac{1}{E-E_{n}-i \varepsilon}-\frac{1}{E-E_{n}+i \varepsilon}\right\}= \\
& =\pi \sum_{n} \delta\left(E-E_{n}\right)=\pi \operatorname{Tr} \delta(E-\mathbf{H})=\operatorname{Im} R(E) .
\end{aligned}
$$

Таким образом, мы хотим исключить из рассмотрения ненужные вклады ${ }^{1)}$ с $E \neq E_{n}$, содержашиеся именно в $\operatorname{Re}\left\{1 /\left(E-E_{n}-i \varepsilon\right)\right\}$.

Можно показать [4], что

$$
\rho(E) \sim \sum_{k=-\infty}^{+\infty} e^{i k\left(S_{1}\left(u_{\mathrm{c}}\right)-\pi\right)}=2 \pi \sum_{n} \delta\left(S_{1}\left(u_{\mathrm{c}}\right)-(2 n+1) \pi\right) .
$$

Таким образом, так же как в квазиклассическом случае (2.2), в квантовой теории возмущений необходимо найти поправки к фазе $S_{1}\left(u_{c}\right)$, т.е. вычислить эффективное действие.

В терминах интегралов вышеописанное свойство отсутствия (“сокрашения") реальной части при вычислении квадрата модуля (см. (2.3)) выглядит следуюшим образом. Рассмотрим выражение

$$
\begin{aligned}
\rho(E) & =\sum_{n}\left|\frac{1}{E-E_{n}-i \varepsilon}\right|^{2}= \\
& =\sum_{n} \int_{0}^{\infty} d T_{+} d T_{-} \exp \left\{-\varepsilon\left(T_{+}+T_{-}\right)+i\left(E-E_{n}\right)\left(T_{+}-T_{-}\right)\right\} .
\end{aligned}
$$

Чтобы увидеть эффект сокрашения, введем новые переменные $T$ и $\tau$ :

$$
T_{ \pm}=T \pm \tau,
$$

при этом $0 \leqslant T \leqslant \infty$ и $-T \leqslant \tau \leqslant T$. Однако при $\varepsilon \rightarrow 0$ сушественны значения $T=\left(T_{+}+\right.$ $\left.T_{-}\right) / 2 \sim(1 / \varepsilon) \rightarrow \infty$. По этой причине можно считать, что $|\tau| \leqslant \infty$. В результате получаем

$$
\varepsilon \rho(E)=2 \pi \varepsilon \int_{0}^{\infty} d T e^{2 \varepsilon T} \int_{-\infty}^{+\infty} \frac{d \tau}{\pi} e^{2 i\left(E-E_{n}\right) \tau} .
$$

В последнем интеграле все вклады с $E \neq E_{n}$ сокрашаются, а при $E=E_{n}$ этот интеграл равен бесконечности.

Описанное сокрашение не случайно и не является приближенным, т.к. отражает условие унитарности. Мы увидим, что $\delta$-функция в мере (1.2) имеет ту же природу, что и $\delta$-функция в правой части $(2.3)$, т.е. $\delta$-образная мера возникает при вычислении абсорбционной части.

Отметим, что мы вынуждены начинать с вычисления модуля амплитуд, т.к. известно интегральное представление лишь для амплитуд. Далее мы используем условие унитарности, чтобы найти меру абсорбционной части, которая оказывается $\delta$-образной.

\footnotetext{
1) Термин "ненужные" означает для нас не измеримые в данном эксперименте величины.
} 
2.2. Функциональная $\delta$-образная мера. Чтобы вычислить функцию

$$
\rho(E)=\int d u_{1} d u_{2}\left|a\left(u_{1}, u_{2} ; E\right)\right|^{2},
$$

воспользуемся интегральным представлением

$$
\begin{aligned}
a\left(u_{1}, u_{2} ; E\right) & =i \int_{0}^{\infty} d T e^{i E T} \int_{u(0)=u_{1}}^{u(T)=u_{2}} D u e^{i S_{C_{+}(T)}(u)}, \\
D u & =\prod_{t \in C_{+}(T)} \frac{d u(t)}{\sqrt{2 \pi}} .
\end{aligned}
$$

Здесь и везде в дальнейшем действие $S_{C_{+}(T)}(u)$ определено на комплексном временном контуре Миллса [9]

$$
C_{ \pm}(T): t \rightarrow t \pm i \varepsilon, \quad \varepsilon \rightarrow+0, \quad 0 \leqslant t \leqslant T .
$$

Подставив (2.9) в (2.8), найдем

$$
\begin{aligned}
\rho(E)= & \int_{0}^{\infty} d T_{+} d T_{-} e^{i E\left(T_{+}-T_{-}\right)} \times \\
& \times \int_{u_{+}(0)=u_{-}(0)}^{u_{+}\left(T_{+}\right)=u_{-}\left(T_{-}\right)} D u_{+} D u_{-} \exp \left\{i S_{C_{+}\left(T_{+}\right)}\left(u_{+}\right)-i S_{C_{-}\left(T_{-}\right)}\left(u_{-}\right)\right\}
\end{aligned}
$$

Отметим важное для дальнейшего граничное условие "замкнутости траектории"

$$
u_{+}(0)=u_{-}(0), \quad u_{+}\left(T_{+}\right)=u_{-}\left(T_{-}\right),
$$

которое является непосредственным следствием определения функции $\rho(E)$.

Вновь введем переменные $T$ и $\tau(2.6)$. Интеграл по $\tau$ будет вычисляться пертурбативно. В нулевом порядке по $\tau$ условия (2.12) примут вид

$$
u_{+}(0)=u_{-}(0), \quad u_{+}(T)=u_{-}(T) .
$$

Следует отметить, что данное решение системы условий (2.12) единственное, которое не противоречит соотношению неопределенности квантовой механики, если интеграл по $\tau$ вычисляется пертурбативно (другие решения будут содержать тогда ограничения на производные координат по времени).

Если мы введем новые координаты $u$ и $x$ :

$$
u_{ \pm}(t)=u(t) \pm x(t)
$$

то условия (2.13) дают

$$
x(0)=x(T)=0 .
$$

Значения $u(0)$ и $u(T)$ при этом остаются произвольными. Мы увидим, что эта "минимальная" система граничных условий достаточна, чтобы определить интегралы по $\tau$ и $x$. 
Разложим действие по замкнутому пути

$$
S_{\mathrm{cl}}(u \pm x ; T \pm \tau) \equiv\left(S_{C_{+}(T+\tau)}(u+x)-S_{C_{-}(T-\tau)}(u-x)\right)
$$

в ряд по степеням $\tau$

$$
S_{\mathrm{cl}}(u \pm x ; T \pm \tau)=S_{\mathrm{cl}}(u \pm x ; T)-2 \tau H_{T}(u)-2 \widetilde{H}_{T}(u ; \tau)
$$

где гамильтониан в момент времени $T$ :

$$
H_{T}(u)=-\frac{\partial}{\partial T} S_{C_{+}(T)}(u)
$$

не зависит от $x$ в силу условий $(2.15)$. Остаток разложения $\widetilde{H}_{T}(u ; \tau)$ содержит высшие порядки по $\tau$ :

$$
\widetilde{H}_{T}(u ; \tau)=\sum_{n=1}^{\infty} \frac{\tau^{2 n+1}}{(2 n+1) !} \frac{d^{2 n}}{d T^{2 n}} H_{T}(u) .
$$

Следовательно, условия (2.15) факторизуют зависимость от $\tau$ и $x(t)$ : зависимость от $x$ содержится лиш в не зависяшей от $\tau$ величине $S_{\mathrm{cl}}(u \pm x ; T)$. Поэтому мы можем построить теорию возмушений по $\tau$ и $x$ независимо.

Рассмотрим теперь разложение по степеням $x$ :

$$
S_{\mathrm{cl}}(u \pm x ; T)=S_{P(T)}(u)-2 \operatorname{Re} \int_{C_{+}(T)} d t x(t) \frac{\delta S_{C_{+}}(u)}{\delta u(t)}-2 \widetilde{V}_{T}(u, x)
$$

где первый член разложения

$$
S_{P(T)}(u)=\left(S_{C_{+}(T)}(u)-S_{C_{-}(T)}(u)\right)
$$

Если движение периодическое, то $S_{P(T)}(u)$ не равно тож дественно нулю даже на действительной временной оси [4]. В квазиклассическом приближении величина

$$
S_{P}(T)\left(u_{\mathrm{c}}\right)=k S_{1}\left(u_{\mathrm{c}}\right), \quad k=0, \pm 1, \ldots,
$$

и не зависит от $T$. Причину этого мы покажем в п. 3.3 .

Как обычно,

$$
2 \operatorname{Re} \int_{C_{+}} d t=\int_{C_{+}} d t+\int_{C_{-}} d t
$$

т.к. для произвольной аналитической функции имеет место равенство $f\left(t \in C_{+}\right)=$ $f^{*}\left(t \in C_{-}\right)$.

Нам будет полезен следующий формальный прием. Можно написать, что

$$
\exp \left\{-2 i \widetilde{H}_{T}(u ; \tau)\right\}=\sum_{n} \frac{\tau^{n}}{n !} b_{n}(u ; T)
$$


где

$$
b_{n}(u ; T)=\left.\frac{d^{n}}{d \tau_{1}^{n}} e^{-2 i H_{T}\left(u ; \tau_{1}\right)}\right|_{\tau_{1}=0} \equiv \hat{\tau}_{1}^{n} e^{-2 i \widetilde{H}_{T}\left(u ; \tau_{1}\right)} .
$$

С другой стороны,

$$
(2 i \tau)^{n}=\left.\frac{d^{n}}{d \varepsilon^{n}} e^{2 i \varepsilon \tau}\right|_{\varepsilon=0} \equiv \hat{\varepsilon}^{n} e^{2 i \varepsilon \tau}
$$

Таким образом,

$$
\begin{aligned}
\exp \left\{-2 i \widetilde{H}_{T}(u ; \tau)\right\} & =\sum_{n} \frac{1}{n !}\left(\frac{\hat{\tau}_{1} \hat{\varepsilon}}{2 i}\right)^{n} e^{2 i \varepsilon \tau} \exp \left\{-2 i \widetilde{H}_{T}\left(u ; \tau_{1}\right)\right\}= \\
& =\exp \left\{-\frac{i \hat{\tau}_{1} \hat{\varepsilon}}{2}\right\} e^{2 i \varepsilon \tau} \exp \left\{-2 i \widetilde{H}_{T}\left(u ; \tau_{1}\right)\right\}
\end{aligned}
$$

Разложение оператора $e^{-i \hat{\tau}_{1} \hat{\varepsilon} / 2}$ будет генерировать соответствующую теорию возмушений.

Такой же оператор возникнет в разложении по локальной величине $x$ :

$$
\begin{aligned}
\exp \left\{-2 i \widetilde{V}_{T}(u, x)\right\}= & \exp \left\{-\frac{i}{2} \operatorname{Re} \int_{C_{+}} d t \hat{j}(t) \hat{x}_{1}(t)\right\} \times \\
& \times \exp \left\{2 i \operatorname{Re} \int_{C_{+}} d t j(t) x(t)\right\} \exp \left\{-2 i \widetilde{V}_{T}\left(u, x_{1}\right)\right\}
\end{aligned}
$$

Отметим, что равенства $(2.21),(2.22)$ линеаризуют аргументы соответствующих экспонент. Тогда, используя соотношения $(2.16),(2.18),(2.21)$ и (2.22), мы найдем, что

$$
\begin{aligned}
\rho(E)= & 2 \pi \int_{0}^{\infty} d T e^{-i \mathbf{K}(\varepsilon \tau, j x)} \int D M(u) \delta\left(E+\varepsilon-H_{T}(u)\right) \times \\
& \times \exp \left\{i S_{P(T)}(u)\right\} \exp \left\{-2 i \widetilde{H}_{T}(u ; \tau)-2 i \widetilde{V}_{T}(u, x)\right\},
\end{aligned}
$$

где разложение по степеням оператора

$$
\mathbf{K}=\frac{1}{2}\left(\hat{\tau} \hat{\varepsilon}+\operatorname{Re} \int_{C_{+}} d t \hat{j}(t) \hat{x}(t)\right)=\frac{1}{2}\left(\frac{\partial}{\partial \tau} \frac{\partial}{\partial \varepsilon}+\operatorname{Re} \int_{C_{+}} d t \frac{\delta}{\delta j(t)} \frac{\delta}{\delta x(t)}\right)
$$

дает ряд теории возмущений. Все вспомогательные переменные $\tau, \varepsilon, j, x$ должны быть взяты равными нулю в конце вычислений.

Мера в (2.23) определена соотношением

$$
D M(u)=\prod_{t} d u \delta\left(\frac{\delta S(u)}{\delta u}+j\right)=\prod_{t} d u \delta\left(\ddot{u}+v^{\prime}(u)-j\right),
$$

а $\delta$-рункция - равенством

$$
\prod_{t} \delta\left(\ddot{u}+v^{\prime}(u)-j\right)=\int_{x(0)=0}^{x(T)=0} \prod_{t} \frac{d x}{\pi} \exp \left\{2 i \operatorname{Re} \int d t x\left(\ddot{u}+v^{\prime}(u)-j\right)\right\} .
$$


Аргумент $\delta$-функции не содержит граничных значений $u(0)$ и $u(T)$. Однако это несушественно, поскольку, чтобы решить уравнение второго порядка (2.28) (см. ниже), необходимо задать две постоянные интегрирования (ими могут быть $u(0)$ и $u(T)$ ); интегрирование по этим постоянным подразумевается.

Экспонента в формуле (2.26) равна

$$
\operatorname{Re} x \operatorname{Re}\left(\ddot{u}+v^{\prime}(u)-j\right)+\operatorname{Im} x \operatorname{Im}\left(\ddot{u}+v^{\prime}(u)-j\right),
$$

поскольку определена на комплексном временном контуре. Это означает, что

$$
\prod_{t} \delta\left(\ddot{u}+v^{\prime}(u)-j\right)=\prod_{t \in C} \delta\left(\operatorname{Re}\left\{\ddot{u}+v^{\prime}(u)-j\right\}\right) \delta\left(i \operatorname{Im}\left\{\ddot{u}+v^{\prime}(u)-j\right\}\right),
$$

где $C=C_{+}+C_{-}$. Следовательно, мера (2.25) определяет как действительную, так и мнимую части решений уравнения (2.28) (см. ниже). Предполагается при этом, что принадлежность аргумента контуру $C_{+}$или $C_{-}$определяет соответствуюшее " $i \varepsilon$-предписание" (см. далее решение уравнения (2.31) в следуюшем пункте).

Величина $\ddot{u}+v^{\prime}(u)-j$ является полной силой, и поэтому произведение $\left(\ddot{u}+v^{\prime}(u)-j\right) x$ есть виртуальная работа. В классической механике эта работа равна нулю, т.к. классическая механика обратима во времени (Даламбер). В результате, поскольку виртуальное отклонение произвольно, мы находим локальное условие

$$
\ddot{u}+v^{\prime}(u)-j=0
$$

обратимости механического движения во времени.

В квантовой механике виртуальная работа не равна нулю (квантовые поправки сдвигают уровни энергии), но интеграл по $x(t)$ дает тот же результат (2.28). Мы можем заключить, что условие унитарности квантовых систем позволяет вывести вариационный принцип Даламбера [4] (см. также [10]).

2.3. Свойства теории с $\delta$-образной мерой. Уравнение (2.28) может быть решено с помошью разложения по $j(t)$ :

$$
u_{j}(t)=u_{\mathrm{c}}(t)+\int d t^{\prime} G\left(t, t^{\prime} ; u_{c}\right) j\left(t^{\prime}\right)+\text { высшие поправки по } j,
$$

где $u_{\mathrm{c}}$ - решение однородного уравнения

$$
\ddot{u}+v^{\prime}(u)=0
$$

и $G\left(t, t^{\prime} ; u_{\mathrm{c}}\right)$ - функция Грина,

$$
\left(\partial_{t}^{2}+v^{\prime \prime}\left(u_{\mathrm{c}}\right)\right) G\left(t, t^{\prime} ; u_{\mathrm{c}}\right)=\delta\left(t-t^{\prime}\right) .
$$

При этом, если $\left(t, t^{\prime}\right) \in C_{+}$, то решение этого уравнения $G^{\mathrm{F}}\left(t, t^{\prime} ; u_{\mathrm{c}}\right)$ есть каузальная (фейнмановская) функция Грина. Если $\left(t, t^{\prime}\right) \in C_{-}$, то решением будет $G^{\mathrm{F}}\left(t, t^{\prime} ; u_{\mathrm{c}}\right)^{*}$ антикаузальная функция Грина. 
Отметим также, что оператор $\left(\partial_{t}^{2}+v^{\prime \prime}\left(u_{\mathrm{c}}\right)\right)$ трансляционно-неинвариантен. По этой причине рассматриваемая схема теории возмущений чрезвычайно сложна ${ }^{2}$, что и было основным препятствием для ее дальнейшего развития.

При нашем выборе потенциала уравнение (2.30) имеет как "тривиальное" решение

$$
u_{0}: \dot{u}_{0}=0, \quad v^{\prime}\left(u_{0}\right)=0
$$

так и нетривиальное

$$
u_{\mathrm{c}}=u_{\mathrm{c}}(t): \dot{u}_{\mathrm{c}}(t) \neq 0, \quad \ddot{u}_{c}+v^{\prime}\left(u_{c}\right) \equiv 0 .
$$

В силу определения $\delta$-функции, а также отсутствия специальных ограничений на класс возможных решений оба перечисленных выше решения должны быть учтены, т.е.

$$
\rho(E)=\rho_{0}(E)+\rho_{\mathrm{c}}(E) .
$$

Это означает, что мы должны просуммировать по всем возможным для данной задачи топологическим классам траекторий, если граничные условия таковы, что один класс не может покрыть весь фазовый объем. Каждый класс траекторий принадлежит ограниченной области фазового пространства. В нашем случае $\Omega=W_{0}+W_{\mathrm{c}}$. Каждая подобласть $W_{i}$ ограничена линией бифуркации [12]. Это означает, что $\rho_{0}$ не может быть получено аналитическим продолжением $\rho_{\mathrm{c}}$ (например, путем выбора $E=0$ в $\rho_{\mathrm{c}}$ ).

Очевидно, что мы должны оставить в сумме (2.34) вклад, которому соответствует наибольший по величине объем $V_{W_{i}}, i=0$, с. Это отвечает тому, что мы оставляем вклад траектории $u_{\mathrm{c}} \in W_{\mathrm{c}}$ и отбрасываем вклад $u_{0}$, т.к. соответствуюшая подобласть $W^{0}$ является точкой $[4,12]$. Действительно, будет показано, что $\rho_{\mathrm{c}} \sim V_{\mathrm{tr}}=\infty$, где $V_{\mathrm{tr}}=$ $V_{W_{0}}$-объем трансляционной (нулевой) моды, при этом $\rho_{0} \sim O(1)$. Следовательно, если трансляционная симметрия не нарушена, можно сказать, что $\rho_{0}$ реализуется на мере порядка $O(1) / V_{\mathrm{tr}}=0$.

Топологическая классификация вкладов оказывается возможной из-за отсутствия в решении (2.34) интерференции вкладов $u_{0}$ и $u_{\mathrm{c}}$, что отражает ортогональность соответствуюших гильбертовых пространств. Соответственно сформулированное выше правило отбора описывает наше правило выбора основного состояния.

\section{3. ТЕОРИЯ ВОЗМУЩЕНИЙ ВКБ}

Можно показать, что соотношение (2.23) восстанавливает обычную схему теории возмушений по степеням $g$. Для этого, во-первых, надо найти решение неоднородного уравнения (2.28) (см. ниже п. 3.1). Во-вторых, необходимо установить, что эта теория возмушений представляет собой каноническое разложение по степеням $g$ (см. ниже п. 3.2). В п. 3.3 мы покажем, как выглядит проблема нулевых мод в теории с $\delta$-образной мерой.

\footnotetext{
${ }^{2)}$ Импульсное приближение при решении уравнения (2.31) было рассмотрено в статье [11].
} 
3.1. Древесное разложение. Рассмотрим потенциал вида

$$
v(u ; g)=\frac{1}{2} w_{0}^{2} u^{2}+\frac{1}{4} g u^{4}
$$

и “древесное" разложение (2.29) для этого потенциала. Очевидно, что

$$
v(u ; g)=\frac{1}{g} v\left(g^{\frac{1}{2}} u\right) .
$$

Разложение (2.29) может быть записано в виде

$$
u_{j}(t)=u_{\mathrm{c}}(t)+\sum_{n=1}^{\infty} \frac{1}{n !} \int \prod_{k=1}^{n}\left\{d t_{i} j\left(t_{i}\right)\right\} G_{n}\left(t, t_{1}, \ldots, t_{n} ; u_{\mathrm{c}}\right) .
$$

Достаточно просто показать, что $n$-точечная функция Грина [1]

$$
G_{n}=O\left(g^{\frac{n-1}{2}}\right)
$$

Действительно, подставив (3.3) в уравнение

$$
\ddot{u}+\omega_{0}^{2} u+g u^{3}=j
$$

найдем, что

$$
\left(\partial_{t}^{2}+\omega_{0}^{2}+3 g u_{\mathrm{c}}^{2}\right) G_{1}\left(t, t_{1} ; u_{\mathrm{c}}\right)=\delta\left(t-t_{1}\right) .
$$

Оператор $\left(\partial_{t}^{2}+\omega^{2}+3 g u_{\mathrm{c}}^{2}\right)$ не зависит от $g$, т.к. $u_{c}=O\left(1 / g^{1 / 2}\right)$. Следовательно $G_{1}=$ $O\left(g^{0}\right)$. Уравнение для $G_{2}$ имеет вид

$$
\left(\partial_{t}^{2}+\omega^{2}+3 g u_{\mathrm{c}}^{2}\right) G_{2}\left(t, t_{1}, t_{2} ; u_{\mathrm{c}}\right)+6 g u_{\mathrm{c}} G_{1}\left(t, t_{1} ; u_{\mathrm{c}}\right) G_{1}\left(t, t_{2} ; u_{\mathrm{c}}\right)=0 .
$$

Таким образом, в согласии с (3.4) $G_{2}=O\left(g^{1 / 2}\right)$. В результате аналогичного анализа высших порядков по $j$ нетрудно получить оценку (3.4).

Функционал, отвечающий взаимодействию для случая (3.1), имеет вид

$$
\widetilde{V}_{T}(u, x)=2 g \operatorname{Re} \int_{C_{+}} d t x^{3}(t) u(t)+O(\varepsilon),
$$

где $O(\varepsilon)$ пропорционален мнимой части $S_{\mathrm{cl}}$.

Оператор $\mathbf{K}$ линеен по $\hat{x}=\delta / \delta x$. Поэтому действие оператора $\exp \{-i \mathbf{K}\}$ даст

$$
\begin{aligned}
\rho_{\mathrm{c}} \sim & : \exp \left\{-2 i \widetilde{\mathbf{V}}_{T}\left(u, \frac{\hat{j}}{2 i}\right)\right\}: \exp \left\{i S_{P(T)}(u)\right\} \times \\
& \times \exp \left\{-2 i \widetilde{H}_{T}(u ; \tau)\right\} \delta\left(E+\varepsilon-H_{T}\left(u_{j}\right)\right)
\end{aligned}
$$

где двоеточие означает нормальное произведение, когда оператор стоит слева от функций, на которые он может подействовать. Все несущественные для анализа интегралы в 
выражении (3.9) были опушены. Разложение операторной экспоненты приводит к ряду теории возмущений

$$
\rho_{\mathrm{c}} \sim \sum_{n} \frac{(-2 i)^{n}}{n !}: \tilde{\mathbf{V}}_{T}^{n}\left(u_{j}, \frac{\hat{j}}{2 i}\right): e^{i S_{P(T)}\left(u_{j}\right)} e^{-2 i \tilde{H}_{T}\left(u_{j} ; \tau\right)} \delta\left(E+\varepsilon-H_{T}\left(u_{j}\right)\right) .
$$

Рассмотрим сначала члены, отвечающие самодействию. Это означает, что перенормировку $S_{P}$ и $\widetilde{H}_{T}$ на этом этапе мы опускаем, рассматривая вклады, пропорциональные

$$
\delta\left(E+\varepsilon-H_{T}\left(u_{j}\right)\right) e^{i S_{P(T)}\left(u_{c}\right)} e^{-2 i \widetilde{H}_{T}\left(u_{c} ; \tau\right)} \sum_{n} \frac{(-2 i)^{n}}{n !}: \widetilde{\mathbf{V}}_{T}^{n}\left(u_{j}, \frac{\hat{j}}{2 i}\right): .
$$

Тогда в низшем порядке имеем вклад, пропорциональный

$$
\hat{j}^{3} u_{j} \sim G_{3}=O\left(g^{2}\right)
$$

Здесь была использована оценка (3.4) и было учтено, что вспомогательная переменная $j$ должна быть положена равной нулю. Во втором порядке, когда $\widetilde{\mathbf{V}}_{T}^{2}=O\left(\hat{j}^{6}\right)$, мы имеем вклад

$$
g^{2} \hat{j}^{6} u_{j}^{2}=O\left(g^{4}\right)
$$

и т.д. Можно найти в результате, что $n$-й порядок разложения $\exp \{-i \mathbf{K}\}$ пропорционален $O\left(g^{2 n}\right)$.

Как следует из разложения (3.3) и оценки (3.4), действие оператора $\hat{j}$ на $u_{j}$ дает член порядка $g^{1 / 2}$. Тогда перенормировка величин $S_{P}$ и $\widetilde{H}_{T}$, которые в квазиклассическом приближении имеют порядок $1 / g$, обязательно приводит к рядам теории возмушений по положительным степеням $g$, поскольку оператор, генерируюший ряд теории возмушений, пропорционален $\prod \hat{j}\left(t_{i}\right)$.

3.2. Связь с ВКБ-разложением. Можно привести другой аргумент в пользу того, что полученная вьше теория возмушений представляет собой обычное разложение в окрестности $u_{\mathrm{c}}$, которое рассматривалось в ранних публикациях $[1,7]$. Рассмотрим для этой цели подстановку

$$
u(t) \rightarrow u_{\mathrm{c}}(t)+u(t)
$$

Тогда

$$
\begin{aligned}
\rho_{\mathrm{c}}(E)= & 2 \pi \int_{0}^{\infty} d T e^{-i \mathbf{K}(\varepsilon \tau, \mathbf{j} \mathbf{x})} \int D M\left(u_{\mathrm{c}}, u\right) \delta\left(E+\varepsilon-H_{T}\left(u_{\mathrm{c}}+u\right)\right) \times \\
& \times \exp \left\{i S_{P(T)}\left(u_{\mathrm{c}}+u\right)\right\} \exp \left\{-2 i \widetilde{H}_{T}\left(u_{\mathrm{c}}+u ; \tau\right)-2 i \widetilde{V}_{T}\left(u_{\mathrm{c}}+u, x\right)\right\}
\end{aligned}
$$

где

$$
D M\left(u_{\mathrm{c}}, u\right)=\prod_{t} d u \delta\left(\frac{\delta S\left(u_{\mathrm{c}}+u\right)}{\delta u}+j\right)
$$




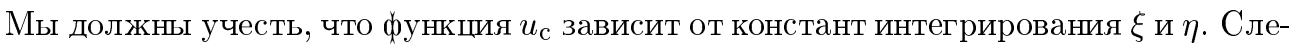
довательно, если пара $(\xi, \eta)$ образует многообразие $W_{\mathrm{c}}$, что отмечалось в п. 2.3 , необходимо в выражении (3.15) в силу того же определения $\delta$-функции провести суммирование по всем значениям $(\xi, \eta)$. Подробно этот вопрос обсуждается ниже в п. 3.3, здесь же мы рассмотрим вклад с конкретным выбором $(\xi, \eta)$.

Мы хотим показать, что представление (3.14) может быть редуцировано к произведению двух интегралов по путям. Действительно, используя соотношения $(2.26),(2.16)$ и (2.18), мы найдем из (3.14), что

$$
\begin{aligned}
\rho_{\mathrm{c}}(E)= & 2 \int_{0}^{\infty} d T \int_{-\infty}^{+\infty} d \tau^{\prime} e^{-i \mathbf{K}} \int D u D x^{\prime} \exp \left\{2 i\left(E+\varepsilon-H_{T}\left(u_{\mathrm{c}}+u\right)\right) \tau^{\prime}\right\} \times \\
& \times \exp \left\{2 i H_{T}\left(u_{\mathrm{c}}+u\right) \tau\right\} \exp \left\{S_{\mathrm{cl}}\left(u_{\mathrm{c}}+u \pm x^{\prime} ; T \pm \tau^{\prime}\right)\right\} \times \\
& \times \exp \left\{2 i \operatorname{Re} \int d t x\left(\frac{\partial S\left(u_{\mathrm{c}}+u\right)}{\partial u}\right)\right\} \times \\
& \times \exp \left\{-2 i \operatorname{Re} \int d t x\left\{\frac{\partial S\left(u_{\mathrm{c}}+u\right)}{\partial u}+j\right\}\right\} .
\end{aligned}
$$

Действие оператора $\exp \{-i \mathbf{K}\}$ приводит к подстановке $x \rightarrow x^{\prime}, \tau \rightarrow \tau^{\prime}$ и $\varepsilon \rightarrow 0, j \rightarrow 0$. Учтя это, найдем

$$
\rho_{\mathrm{c}}(E)=\left|\int_{0}^{\infty} d T e^{i E T} \int D u e^{i S_{C_{+}}\left(u_{\mathrm{c}}+u\right)}\right|^{2},
$$

где при вычислении функционального интеграла необходимо выполнить разложение в окрестности $u=0$. Именно такие интегралы вычислялись в работах $[1,7]$.

3.3. Нулевые моды. Равенство (3.17) для $\rho_{\mathrm{c}}(E)$ требует доопределения, поскольку нет каких-либо физических ограничений на величины $(\xi, \eta)$ : мы должны просуммировать по всем их значениям. Следуя определению

$$
\sum_{\left\{u_{\mathrm{c}}\right\}}=\int_{W_{\mathrm{c}}} d \xi d \eta \sigma\left(u_{\mathrm{c}} ; \xi, \eta\right),
$$

можно считать, что надо найти плотность $\sigma\left(u_{\mathrm{c}} ; \xi, \eta\right)$ в интервале $(\xi, \xi+d \xi ; \eta, \eta+d \eta)$. Для этой цели обычно используется подстановка Фаддеева-Попова [7].

Необходимо различать движение вдоль траектории $u_{\mathrm{c}}$, параметризованное временем $t$, и движение от траектории к траектории за счет изменения параметров $(\xi, \eta)$. Последнее обычно рассматривается как нефизическое (нединамическое) и поэтому должно быть отделено от физического движения вдоль траектории $u_{c}$. Подстановка Фаддеева-Попова выбирается именно с этим расчетом.

По определению величины $(\xi, \eta)$ являются постоянными интегрирования и могут быть выбраны произвольно. Например, $(\xi, \eta)$ могут быть выбраны как начальная координата и импульс частицы на траектории $u_{\mathrm{c}}$.

Однако более перспективно “теоретико-полевое” определение этих величин (см. далее раздел 4). Как обычно, следует отметить, что конкретный выбор $(\xi, \eta)$ означает нарушение симметрии. Тогда величина $\eta$ может быть выбрана как генератор $J$ нарушенной симметрии, а $\xi$ - как канонически-сопряженная координата $\Theta$. Нам будет важно, 
что конкретный выбор $(\xi, \eta)$ однозначно определяет $u_{c}$. Другими словами, мы используем обычное в механике утверждение [12], что пара $(\xi, \eta)$ образует многообразие $W_{\mathrm{c}}$ и функция $u_{\mathrm{c}}$ принадлежит ему полностью. Это означает, что уравнения

$$
\xi=\Theta\left(u_{\mathrm{c}}, \dot{u}_{\mathrm{c}}\right), \quad \eta=J\left(u_{\mathrm{c}}, \dot{u}_{\mathrm{c}}\right)
$$

фиксируют постоянные интегрирования $u_{\mathrm{c}}$ однозначно.

Тогда, чтобы определить плотность $\sigma$, можно подставить в начальное интегральное представление (2.23) единицу (подстановка Фаддеева-Попова), записанную в виде

$$
1=\int_{W_{\mathrm{c}}} \prod_{t} d \xi d \eta \delta(\xi-\Theta(u, \dot{u})) \delta(\eta-J(u, \dot{u})) .
$$

Отметим, что по определению величина $\eta$ совпадает с сохраняюшимся в классической механике генератором. Но в квантовом случае следует полагать $\eta=\eta(t)$.

Теперь можно изменить порядок интегрирования и проинтегрировать по $u$, используя $\delta$-функцию в мере $D M$. Уравнение Лагранжа (2.30) должно быть решено с учетом уравнений (3.18). При этом мера $D M_{\mathrm{c}}$ не зависит от выбора численного значения $\xi$, поскольку уравнения движения инвариантны относительно изменений $\xi$ и $j=j(t)-$ вспомогательная величина, которая может быть выбрана произвольно. Это означает, что функция $\rho_{\mathrm{c}}(E)$ должна быть пропорциональна объему

$$
V_{\mathrm{tr}}=\int d \xi(0)=\int d \xi_{0}
$$

трансляционной по времени моды, поскольку в силу условия унитарности

$$
\rho_{\mathrm{c}}(E) \sim \operatorname{Im} R(E) .
$$

Однако, с другой стороны, как следует из (3.17), можно было бы ожидать, что $\rho_{\mathrm{c}} \sim$ $V_{\mathrm{tr}}^{2}$. Поэтому, чтобы разрешить возникаюшее противоречие, мы должны были вставить единицу Фаддеева-Попова, определенную на полном контуре $C=C_{+}+C_{-}$(см. (2.27)), чтобы учесть полную независимость траекторий $u_{+}(t)$ и $u_{-}(t)$. Это, в свою очередь, означает, что граничные значения этих траекторий различны, т.е. если мы введем переменные $\left.\xi\left(t \in C_{ \pm}\right)\right|_{t=0} \equiv \xi_{ \pm}$, следует учитывать, что, вообше говоря, $\xi_{+} \neq \xi_{-}$. Тогда интегрирование по $\xi_{+}$и $\xi_{-}$должно проводиться раздельно. При этом, однако, следует помнить о единственном ограничении, что траектории $u_{+}$и $u_{-}$должны в совокупности составлять замкнутую траекторию. Это условие необходимо, т.к. лишш в этом случае соотношение $\rho_{\mathrm{c}}(E) \sim \operatorname{Im} R(E)$ выполнимо. Это, в свою очередь, может в зависимости от формы траектории $u_{\mathrm{c}}$ ограничить независимость переменных $\xi_{+}$и $\xi_{-}$.

Нами рассматриваются периодические траектории. Поэтому, учитывая условия $(2.15)$, мы найдем, что траектории $u_{+}(t)$ и $u_{-}(t)$ на концах будут совпадать, если

$$
\xi_{+}=\xi_{-}+k P_{1}(E), \quad k=0, \pm 1, \pm 2, \ldots,
$$

где $P_{1}(E)$ - элементарный период. Именно это обстоятельство приводит к тому, что $S_{P} \neq 0$, а необходимое суммирование по $k$ влечет за собой условие квантования уровней энергии (2.4) (см. также работы $[4,8]$ ). 


\section{4. КАНОНИЧЕСКОЕ ПРЕОБРАЗОВАНИЕ}

Квантовая природа коллективных переменных $(\xi, \eta)$ отмечалась многими авторами $[1,13]$. Мы продолжим эту идею и будем рассматривать коллективные переменные как новые обобшенные координаты. Иными словами, если в каноническом подходе степени свободы $(\xi, \eta)$ рассматриваются как нефизические, которые следует исключить из рассмотрения с помошью подстановки Фаддеева-Попова, в развиваемом подходе именно переменные $(\xi, \eta)$ будут рассматриваться как фундаментальные поля.

Мы используем $\delta$-образность меры, определение индуцируюшего взаимодействия функционала $\widetilde{V}_{T}$ и явный вид генерируюшего ряд теории возмушений оператора $\exp \{\mathbf{K}\}$, чтобы перечислить возможные возмущения поля $u_{\mathrm{c}} \in W_{\mathrm{c}}$ в терминах $(\xi, \eta)$ (см. далее п. 4.1). В п. 4.2 ниже показана структура новой теории возмушений по степеням $1 / g$.

4.1. Процедура отображения. Вернемся к единице Фаддеева-Попова

$$
1=\int D \xi D \eta \prod_{t} \delta(\xi-\Phi(u, \dot{u})) \delta(\eta-J(u, \dot{u})) .
$$

Здесь предполагается (см. п. 3.3), что

$$
\prod_{t} \equiv \prod_{t \in C=C_{+}+C_{-}}
$$

Нам будет удобен также формализм первого порядка. Соответствуюшая мера есть

$$
D M(u, p)=\prod_{t} d u d p \delta\left(\dot{u}-\frac{\partial H_{j}(u, p)}{\partial p}\right) \delta\left(\dot{p}+\frac{\partial H_{j}(u, p)}{\partial u}\right),
$$

где полный гамильтониан

$$
H_{j}(u, p)=\frac{1}{2} p^{2}+v(u)-j u
$$

включает энергию квантовых возмушений $j u$. Ясно, что, проинтегрировав (4.2) по $p$, мы получим исходное выражение (2.25).

Подставив (4.1) в функциональный интеграл с мерой (4.2), мы найдем, что переменные $u$ и $p$ определяются четырьмя уравнениями

$$
\dot{u}=\frac{\partial H_{j}(u, p)}{\partial p}, \quad \dot{p}=-\frac{\partial H_{j}(u, p)}{\partial u}
$$

и

$$
\xi(t)=\Phi(u, \dot{u}), \quad \eta(t)=J(u, \dot{u}) .
$$

В предыдушем разделе мы использовали первую пару уравнений (4.4).

Сейчас же мы используем вторую пару уравнений (4.5). Это имеет смысл тогда и только тогда, когда $u_{\mathrm{c}}$ полностью принадлежит пространству $W_{\mathrm{c}}$ и пространство $W_{\mathrm{c}}$ есть многообразие. Последнее означает, что уравнения (4.5) имеют единственное решение $\left(u_{\mathrm{c}}, p_{\mathrm{c}}\right)$ и это решение преврашает (4.4) в тождество, по крайней мере, при $j=0$. 
Пусть $u_{\mathrm{c}}(\xi, \eta)$ и $p_{\mathrm{c}}(\xi, \eta)$ - решения уравнений $(4.5)$. В этом описании нетрудно узнать обычное каноническое преобразование (1.4), которое определяет расслоение $W_{\mathrm{c}}=T^{*} \Omega$. Но надо помнить, что уравнения (4.4) и (4.5) должны быть решены совместно. Следовательно, подставив $u_{\mathrm{c}}, p_{\mathrm{c}}$ в (4.4), мы должны решить динамическую задачу, описывающую “возбуждение” источником $j$ решений $\xi_{j}(t)$ и $\eta_{j}(t)$.

Таким образом, мы показываем, что случайное (гауссово) блуждание, индуцируемое оператором $\exp \{-i \mathbf{K}\}$, плотно покрывает как пространство $W_{\mathrm{c}}=(\xi, \eta)$, так и пространство $\Omega=(u, p)$. По этой причине неважно, в каком пространстве мы описьваем квантовую динамику.

Якобиан преобразования $\Delta$ вновь $\delta$-образен, т.е.

$$
\Delta=\prod_{t} \delta\left(\dot{u}_{\mathrm{c}}-\frac{\partial H_{j}\left(u_{\mathrm{c}}, p_{\mathrm{c}}\right)}{\partial p_{\mathrm{c}}}\right) \delta\left(\dot{p}_{\mathrm{c}}-\frac{\partial H_{j}\left(u_{\mathrm{c}}, p_{\mathrm{c}}\right)}{\partial u_{\mathrm{c}}}\right)
$$

и

$$
\operatorname{det}^{-1}\left(u_{\mathrm{c}}, p_{\mathrm{c}}\right)=\int \prod_{t} d u d p \delta(\xi-\Phi(u, \dot{u})) \delta(\eta-J(u, \dot{u}))=1,
$$

т.к. преобразование каноническое. Это позволяет диагонализовать преобразование $\Delta$, что дает следующее интегральное представление в пространство $W_{\mathrm{c}}$ :

$$
\begin{aligned}
\rho_{\mathrm{c}}(E)= & 2 \pi \int d T e^{-i \mathbf{K}} \int D M(\xi, \eta) \delta(E+\varepsilon-h(\xi, \eta ; T)) \times \\
& \times \exp \left\{i S_{P(T)}\left(u_{\mathrm{c}}\right)\right\} \exp \left\{-2 i \tilde{h}\left(u_{\mathrm{c}} ; \tau, T\right)-2 i \tilde{V}_{T}\left(u_{\mathrm{c}}, x\right)\right\},
\end{aligned}
$$

где мера

$$
D M(\xi, \eta)=\prod_{t} d \xi d \eta \delta\left(\dot{\xi}-\frac{\partial h_{j}(\xi, \eta)}{\partial \eta}\right) \delta\left(\dot{\eta}+\frac{\partial h_{j}(\xi, \eta)}{\partial \xi}\right)
$$

и $h_{j}$ - преобразованный гамильтониан вида

$$
h_{j}(\xi, \eta)=h(\eta)-j u_{\mathrm{c}}(\xi, \eta)
$$

В результате отображения задача вычисления интеграла свелась к поиску решений уравнений

$$
\begin{aligned}
& \dot{\xi}=\frac{\partial h_{j}(\xi, \eta)}{\partial \eta}=\omega(\eta)-j \frac{\partial u_{\mathrm{c}}(\xi, \eta)}{\partial \eta}, \\
& \dot{\eta}=-\frac{\partial h_{j}(\xi, \eta)}{\partial \xi}=j \frac{\partial u_{\mathrm{c}}(\xi, \eta)}{\partial \xi}
\end{aligned}
$$

При этом удобно выбрать

$$
\omega(\eta)=\frac{\partial h(\eta)}{\partial \eta}=1
$$

Это означает, что

$$
\eta=H(u, p), \quad \xi=\int^{u} \frac{d y}{\sqrt{2(\eta-v(y))}} .
$$

Ясно, что решение этих уравнений определяет функции $u_{\mathrm{c}}(\xi, \eta)$ и $p_{\mathrm{c}}(\xi, \eta)$ однозначно. 
4.2. Структура преобразованной теории возмущений. Покажем теперь, что функция $\rho_{\mathrm{c}}(E)$, определенная в $(4.8)$, имеет разложение сильной связи. Начнем для этого с “древесного разложения" уравнений (4.11):

$$
\dot{\xi}=\frac{\partial h_{j}(\xi, \eta)}{\partial \eta}=1-j \frac{\partial u_{\mathrm{c}}(\xi, \eta)}{\partial \eta}, \quad \dot{\eta}=-\frac{\partial h_{j}(\xi, \eta)}{\partial \xi}=j \frac{\partial u_{\mathrm{c}}(\xi, \eta)}{\partial \xi} .
$$

Мы будем рассматривать следуюшие разложения решений $\xi_{j}$ и $\eta_{j}$ :

$$
\begin{aligned}
& \xi_{j}(t)=\xi_{0}(t)+\sum_{n} \frac{1}{n !} \int \prod_{i=1}^{n}\left\{d t_{i} j\left(t_{i}\right)\right\} \xi_{n}\left(t ; t_{1}, \ldots, t_{n}\right), \\
& \eta_{j}(t)=\eta_{0}(t)+\sum_{n} \frac{1}{n !} \int \prod_{i=1}^{n}\left\{d t_{i} j\left(t_{i}\right)\right\} \eta_{n}\left(t ; t_{1}, \ldots, t_{n}\right) .
\end{aligned}
$$

Подставив разложения (4.15) в уравнения (4.14), мы получим уравнения для $n$-точечных функций Грина $\xi_{n}\left(t ; t_{1}, \ldots, t_{n}\right)$ и $\eta_{n}\left(t ; t_{1}, \ldots, t_{n}\right)$. Можно показать, что

$$
\xi_{n}=O\left(g^{-\frac{n}{2}}\right), \quad \eta_{n}=O\left(g^{-\frac{n}{2}}\right) .
$$

Действительно, в нулевом порядке по $j$ имеем

$$
\xi_{0}=\xi(0)+t, \quad \eta_{0}=\eta(0),
$$

т.к. $W_{\text {c }}$ - однородное и изотропное пространство. В первом порядке по $j$

$$
\begin{aligned}
& \dot{\xi}_{1}\left(t ; t_{1}\right)=-\delta\left(t-t_{1}\right) \frac{\partial u_{\mathrm{c}}\left(\xi_{0}(t), \eta_{0}\right)}{\partial \eta(0)}=O\left(g^{-\frac{1}{2}}\right), \\
& \dot{\eta}_{1}\left(t ; t_{1}\right)=\delta\left(t-t_{1}\right) \frac{\partial u_{\mathrm{c}}\left(\xi_{0}(t), \eta_{0}\right)}{\partial \xi(0)}=O\left(g^{-\frac{1}{2}}\right),
\end{aligned}
$$

т.к. дифференцирование функции $u_{\mathrm{c}}$ по аргументу не может изменить ее зависимость от константы взаимодействия $g$. Во втором порядке мы имеем уравнения, правая часть которых пропорциональна $g^{-1}$,

$$
\begin{aligned}
\dot{\xi}_{2}\left(t ; t_{1}, t_{2}\right)= & -\delta\left(t-t_{1}\right)\left\{\xi_{1}\left(t ; t_{2}\right) \frac{\partial^{2} u_{\mathrm{c}}(t)}{\partial \eta(0) \partial \xi(0)}+\eta_{1}\left(t ; t_{2}\right) \frac{\partial^{2} u_{\mathrm{c}}(t)}{\partial^{2} \eta(0)}\right\}- \\
& -\delta\left(t-t_{2}\right)\left\{\xi_{1}\left(t ; t_{1}\right) \frac{\partial^{2} u_{\mathrm{c}}(t)}{\partial \eta(0) \partial \xi(0)}+\eta_{1}\left(t ; t_{1}\right) \frac{\partial^{2} u_{\mathrm{c}}(t)}{\partial^{2} \eta(0)}\right\}, \\
\dot{\eta}_{2}\left(t ; t_{1}, t_{2}\right)= & \delta\left(t-t_{1}\right)\left\{\xi_{1}\left(t ; t_{2}\right) \frac{\partial^{2} u_{\mathrm{c}}(t)}{\partial^{2} \xi(0)}+\eta_{1}\left(t ; t_{2}\right) \frac{\partial^{2} u_{\mathrm{c}}(t)}{\partial \xi(0) \partial \eta(0)}\right\}+ \\
& +\delta\left(t-t_{2}\right)\left\{\xi_{1}\left(t ; t_{1}\right) \frac{\partial^{2} u_{\mathrm{c}}(t)}{\partial^{2} \xi(0)}+\eta_{1}\left(t ; t_{1}\right) \frac{\partial^{2} u_{\mathrm{c}}(t)}{\partial \xi(0) \partial \eta(0)}\right\}
\end{aligned}
$$

и т.д. Мы видим, что каждый порядок по $\hat{j}$ добавляет множитель $g^{-1 / 2}$, что доказывает оценку (4.16). Важно отметить, что уравнения (4.19) элементарно интегрируемы, 
и мы можем вычислить в отличие от теории возмушений ВКБ величины $\left(\xi_{n}, \eta_{n}\right)$ для произвольных $n$.

Оператор $\mathbf{K}$ линеен по $\hat{x}$. Поэтому

$$
\begin{aligned}
\rho_{\mathrm{c}}(E) \sim & : \exp \left\{-2 i \widetilde{\mathbf{V}}_{T}\left(u_{\mathrm{c}}, \frac{\hat{j}}{2 i}\right)\right\}: \exp \left\{i S_{P(T)}\left(u_{\mathrm{c}}\right)\right\} \times \\
& \times \exp \left\{-2 i \widetilde{H}_{T}\left(u_{\mathrm{c}} ; \tau\right)\right\} \delta\left(E+\varepsilon-h\left(\xi_{j}, \eta_{j} ; T\right)\right) .
\end{aligned}
$$

В конце всех вычислений вспомогательные величины следует положить равными нулю.

Рассмотрим вновь $g u^{4}$-теорию. Тогда

$$
\widetilde{V}_{T}\left(u_{\mathrm{c}}, \frac{\hat{j}}{2 i}\right)=O\left(\hat{j}^{3}\right)
$$

Следовательно, рассматривая только часть, отвечающую самодействию, в низшем порядке будем иметь

$$
\widetilde{V}_{T}\left(u_{\mathrm{c}}, \frac{\hat{j}}{2 i}\right) \sim g \hat{j}^{3} u_{\mathrm{c}}=O\left(\frac{1}{g}\right) .
$$

При этом были использованы соотношения (4.16). Нетрудно видеть, что $n$-й порядок пропорционален $\widetilde{V}_{T}\left(u_{\mathrm{c}}, \hat{j} / 2 i\right)^{n} \sim 1 / g^{n}$.

Действие $\tilde{V}_{T}^{n}\left(u_{\mathrm{c}}, \hat{j} / 2 i\right)$ на величину $e^{i S_{P(T)}\left(u_{\mathrm{c}}\right)} e^{-2 i \widetilde{H}_{T}\left(u_{\mathrm{c}} ; \tau\right)} \delta\left(E+\varepsilon-h\left(\xi_{j}, \eta_{j} ; T\right)\right)$ не изменит структуру ряда, т.к. дифференцирование функции $u_{\mathrm{c}}$ не может изменить ее зависимость от $g$.

\section{5. ЗАКЛЮЧЕНИЕ}

Мы хотим завершить эту работу замечанием, что преобразованное представление $\rho_{\mathrm{c}}$ невозможно свести к представлению в терминах амплитуд типа (3.17). Действительно, вернемся к формуле (4.8) и воспользуемся преобразованием Фурье для наших $\delta$-функций. Тогда

$$
\begin{aligned}
\rho_{\mathrm{c}}(E)= & \int_{0}^{\infty} d T \int_{-\infty}^{+\infty} d \tau^{\prime} e^{-i \mathbf{K}} \int D \xi D \eta D x_{\xi} D x_{\eta} e^{2 i(E+\varepsilon-h(\eta ; T)) \tau^{\prime}} \times \\
& \times \exp \left\{-2 i \operatorname{Re} \int_{C_{+}} d t x_{\xi}\left\{\dot{\xi}-\frac{\partial h_{j}(\xi, \eta)}{\partial \eta}\right\}\right\} \times \\
& \times \exp \left\{-2 i \operatorname{Re} \int_{C_{+}} d t x_{\eta}\left\{\dot{\eta}+\frac{\partial h_{j}(\xi, \eta)}{\partial \xi}\right\}\right\} \times \\
& \times \exp \left\{i S_{P(T)}\left(u_{\mathrm{c}}\right)\right\} \exp \left\{-2 i \tilde{h}\left(u_{\mathrm{c}} ; \tau, T\right)-2 i \widetilde{V}_{T}\left(u_{\mathrm{c}}, x\right)\right\}
\end{aligned}
$$

где (см. разложение $(2.16))$

$$
-2 \tilde{h}\left(u_{\mathrm{c}} ; \tau, T\right)=S_{\mathrm{cl}}\left(u_{\mathrm{c}} \pm x ; T \pm \tau\right)-S_{\mathrm{cl}}\left(u_{\mathrm{c}} \pm x ; T\right)+2 \tau h(\eta) .
$$

4 Теоретическая и математическая физика, т. 123, № 3, 2000 г. 
Помня, что действие оператора $\exp \{-i \hat{e} \hat{\tau} / 2\}$ дает $\tau=\tau^{\prime}$ и $\varepsilon=0$, мы найдем, что

$$
\begin{aligned}
\rho_{\mathrm{c}}(E)= & \int_{0}^{\infty} d T \int_{-\infty}^{+\infty} d \tau e^{2 i E \tau} \exp \left\{-i \operatorname{Re} \int_{C_{+}} d t \frac{\hat{j} \hat{x}}{2}\right\} \int D \xi D \eta D x_{\xi} D x_{\eta} \times \\
& \times \exp \left\{i S_{\mathrm{cl}}\left(u_{\mathrm{c}} \pm x ; T \pm \tau\right)\right\} \exp \left\{-i S_{\mathrm{cl}}\left(u_{\mathrm{c}} \pm x ; T\right)\right\} \times \\
& \times \exp \left\{i S_{P(T)}\left(u_{\mathrm{c}}\right)\right\} \exp \left\{-2 i \widetilde{V}_{T}\left(u_{\mathrm{c}}, x\right)\right\} \times \\
& \times \exp \left\{-2 i \operatorname{Re} \int_{C_{+}} d t x_{\xi} \frac{\delta S\left(u_{\mathrm{c}}\right)}{\delta \eta}\right\} \exp \left\{2 i \operatorname{Re} \int_{C_{+}} d t x_{\eta} \frac{\delta S\left(u_{\mathrm{c}}\right)}{\delta \xi}\right\}
\end{aligned}
$$

если преобразованное действие имеет вид

$$
S\left(u_{\mathrm{c}}\right)=\int d t\{\eta \dot{\xi}-h(\eta)\} .
$$

Подействовав оператором, генерируюшим возмушения, получим с помошью (2.18), что

$$
\begin{aligned}
\rho_{\mathrm{c}}(E)= & \int_{0}^{\infty} d T \int_{-\infty}^{+\infty} d \tau e^{2 i E \tau} \int D \xi D \eta D x_{\xi} D x_{\eta} e^{i S_{\mathrm{cl}}\left(u_{\mathrm{c}} \pm x_{\mathrm{c}} ; T \pm \tau\right)} \times \\
& \times \exp \left\{-2 i \operatorname{Re} \int_{C_{+}} d t\left\{x_{\xi} \frac{\delta S\left(u_{\mathrm{c}}\right)}{\delta \eta}-x_{\eta} \frac{\delta S\left(u_{\mathrm{c}}\right)}{\delta \xi}\right\}\right\} \times \\
& \times \exp \left\{-2 i \operatorname{Re} \int_{C_{+}} d t x_{\mathrm{c}} \frac{\delta S\left(u_{\mathrm{c}}\right)}{\delta u_{\mathrm{c}}}\right\} .
\end{aligned}
$$

При этом были введены локальные координаты

$$
x_{\mathrm{c}}=x_{\xi} \frac{\partial u_{\mathrm{c}}}{\partial \eta}-x_{\eta} \frac{\partial u_{\mathrm{c}}}{\partial \xi}=\delta u_{\mathrm{c}} \wedge \delta p_{\mathrm{c}} .
$$

Теперь, если учесть, что

$$
\frac{\delta S\left(u_{\mathrm{c}}\right)}{\delta \xi}=\frac{\partial u_{\mathrm{c}}}{\partial \xi} \frac{\delta S\left(u_{\mathrm{c}}\right)}{\delta u_{\mathrm{c}}}, \quad \frac{\delta S\left(u_{\mathrm{c}}\right)}{\delta \eta}=\frac{\partial u_{\mathrm{c}}}{\partial \eta} \frac{\delta S\left(u_{\mathrm{c}}\right)}{\delta u_{\mathrm{c}}},
$$

то

$$
\rho_{\mathrm{c}}(E)=\int_{0}^{\infty} d T \int_{-\infty}^{+\infty} d \tau e^{2 i E \tau} \int D \xi D \eta D x_{\xi} D x_{\eta} e^{i S_{\mathrm{cl}}\left(u_{\mathrm{c}} \pm x_{\mathrm{c}} ; T \pm \tau\right)} .
$$

Действие по замкнутому пути есть

$$
\begin{aligned}
S_{\mathrm{cl}}\left(u_{\mathrm{c}} \pm x_{\mathrm{c}} ; T \pm \tau\right)= & S_{C_{+}(T+\tau)}\left(u_{\mathrm{c}}(\xi, \eta)+x_{\mathrm{c}}\left(\xi, \eta ; x_{\xi}, x_{\eta}\right)\right)- \\
& -S_{C_{-}(T-\tau)}\left(u_{\mathrm{c}}(\xi, \eta)-x_{\mathrm{c}}\left(\xi, \eta ; x_{\xi}, x_{\eta}\right)\right) .
\end{aligned}
$$

Отсюда непосредственно видно, что преобразованное выражение не может быть записано в факторизованном виде произведения амплитуд. Мы интерпретируем это заключение как свидетельство о невозможности делать канонические преобразования в интеграле по путям (2.8), поскольку на кокасательных пространствах квантовые возбуждения индуцируют фазовые потоки, в которых все степени свободы перемешаны. 
В традиционных терминах вышесказанное означает наличие проблемы временно́го упорядочения нелинейных операторов. Наш успех в решении этой проблемы основан на наблюдении, что условие унитарности полностью определяет теорию возмушений в (“линейном") представлении, когда мы можем "распутать" все временные упорядочения. Зафиксировав эту процедуру в структурах $D M, \mathbf{K}$ и $\widetilde{V}_{T}$, мы можем перейти далее к любым удобным переменным. Однако “платой” за этот успех является необходимость работать в менее привычном представлении абсорбционных частей амплитуд, и по этой причине следует быть осторожным в утверждении об общности описанного подхода (этот вопрос довольно подробно обсуждается в работе [3]).

Благодарности. В заключение мы хотим поблагодарить В. Г. Кадышевского за интерес к работе.

\section{Список литературы}

[1] Extended Systems in Field Theory. Phys. Rep. 1976. V. 23. № 3. P. 237-374; R. Jackiw. Rev. Mod. Phys. 1977. V. 49. P. 681.

[2] A. Neveu. Phys. Rep. 1976. V. 23. Р. 265; Л.Д. Фаддеев. ТМФ. 1969. Т. 1. С. 3; J. L. Gervais, B. Sakita. Phys. Rev. D. 1975. V. 11. P. 1486.

[3] J. Manjavidze, A. Sissakian. Fields topology and observables. hep-th/9811160.

[4] И. Д. Манджавидзе. ЯФ. 1987. Т. 45. С. 707.

[5] S. F. Edwards, Y. V. Gulyaev. Proc. Roy. Soc. A. 1964. V. 279. P. 229.

[6] K. Abraham, S. Marsden. Foundations of Mechanics. Reading, Mass.: Benjamin/Cummings, 1978; В. И. Арнольд. Математические методы классической механики. М.: Наука, 1989.

[7] J.-L. Gervais, A. Jevicki. Nucl. Phys. B. 1976. V. 110. P. 93; N. H. Christ, T. D. Lee. Phys. Rev. D. 1975. V. 12. P. 1606; J. Godstone, R. Jackiw. Phys. Rev. D. 1975. V. 11. P. 1486; A. Hosoia, K. Kikkawa. Nucl. Phys. B. 1975. V. 101. P. 271.

[8] R. Dashen, B. Hasslacher, A. Neveu. Phys. Rev. D. 1974. V. 10. P. 4114, 4130, 4138; 1975. V. 11. P. 3424.

[9] R. Mills. Propagators of Many-Particles Systems. New York: Gordon \& Breach, 1969.

[10] В. Фож. Вестн. ЛГУ. 1959. Т. 16. С. 442.

[11] B.M. Barbashov, S.P. Kuleshov, V.A. Matveev, V.N. Pervushin, A.N. Sissakian, A. N. Tavkhelidze. Phys. Lett. B. 1970. V. 33. P. 484.

[12] S. Smale. Invent. Math. 1970. V. 11. № 1. P. 45.

[13] В. Е. Корепин, Л. Д. Фаддеев. ТМФ. 1975. Т. 25. С. 147; Л. Д. Фаддеев. Гамильтонова интерпретация метода обратного преобразования рассеяния. В сб.: Солитоны. Ред. Р. Буллаф,, Ф. Кодри. М.: Мир, 1983. С. 363. 